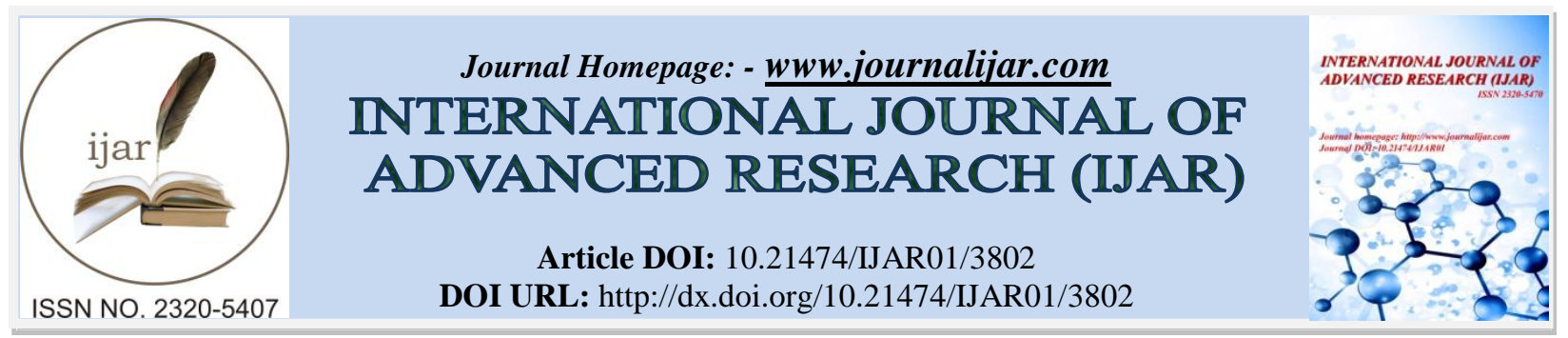

RESEARCH ARTICLE

\title{
PHOTO-CATALYTIC BEHAVIOR OF CDSE QDS SENSITIZED ZR-(1,3,5-BENZENE TRICARBOXYLIC ACID) METAL-ORGANIC FRAMEWORKS
}

Haritha Gonthina ${ }^{1} *$, Gosipathala Sreedhar ${ }^{2}$, and B. Venkateswara Rao ${ }^{1}$.

1. Department of Engineering Chemistry, A U College of Engineering (A), Andhra University, Visakhapatnam530003, India.

2. Electropyro Metallurgy Division, CSIR-Central Electrochemical Research Institute, Karaikudi-630003, Tamilnadu, India.

\section{Manuscript Info}

[.........................

Manuscript History

Received: 19 February 2017

Final Accepted: 10 March 2017

Published: April 2017

Key words:-

Photo-catalysis, ZrBTC Metal-organic

frameworks, CdSe Quantum dots,

Heterostructures, Visible light

illumination, Phloxine B.

\section{Abstract}

Here we reported the synthesis of CdSe quantum dots (QDs) sensitized $\mathrm{Zr}$-1,3,5-benzene tricarboxylic acid (ZrBTC) metal-organic frameworks (MOFs) hetero-structure. The photo-catalytic activity of CdSe QDsZrBTC MOFs is examined for Phloxine B dye degradation under visible light illumination. The CdSe semiconductor QDs presence on ZrBTC MOFs is confirmed by x-ray diffraction (XRD), field emission scanning electron microscopy (FESEM) and transition electron microscopy (TEM) characterizations. The XRD spectrum of CdSe QDs demonstrates the cubic crystalline phase for CdSe QDs. The high crystallinity and bulk phase of ZrBTC MOFs is evinced by XRD pattern of ZrBTC. Surface morphological (FESEM and HRTEM) observations realise that ZrBTC MOFs formed into 2D sheets and sensitized with CdSe QDs. The UV-visible absorption spectrum reveals that the CdSe QDs-ZrBTC hetero-structure absorbs the light at $520 \mathrm{~nm}$ wavelength. The 2D ZrBTC MOFs provide platform for CdSe QDs to hinder the effective electron-hole pair recombination under photoexcitation. The photo-catalytic Phloxine B dye degradation experiments reveals that the CdSe QDs-ZrBTC hetero-structure demonstrated enhanced potential to make use of visible light absorption of $\mathrm{CdSe}$ semiconductor quantum dots in the solar spectrum.

Copy Right, IJAR, 2017,. All rights reserved.

\section{Introduction:-}

One of the attractive research fields in recent years is the photo-assisted catalysis using semiconductors as an advanced oxidation process for the elimination of many organic pollutants in wastewater systems[1]. Photo-catalyst based degradation process has been considered as an alternative technique among various physical[2], chemical[3] and biological[4] techniques for water treatment due to its advantages over the traditional techniques, such as simplicity, quick oxidation, high efficiency and no formation of polycyclic products. Using semiconductors such as metal oxides and sulfides, such as $\mathrm{TiO}_{2}[5], \mathrm{ZnO}[6], \mathrm{Fe}_{2} \mathrm{O}_{3}$ [7] and $\mathrm{WO}_{3}[8]$, for photo-catalytic decolorization of pollutants has received much attention because of possible practical applications. In the past decades there has been a growing interest in the synthesis of various sizes and shapes of semiconductor material nanoparticles as doped with different dopants[9-11].

Corresponding Author:- Haritha Gonthina.

Address:- Department of Engineering Chemistry, A U College of Engineering (A), Andhra University, 
Especially, the semiconductor nanocrystals, the so-called quantum dots (QDs), well-known multifunctional materials, are a newly emerging nanomaterial for degradation of pollutants [12-14]. S.Muthulingam et al [12] synthesized carbon quantum dots/N-doped zinc oxide $(\mathrm{CQD} / \mathrm{N}-\mathrm{ZnO})$ photo-catalyst successfully and the excellent efficiency of the photo-catalyst towards degradation of dyes was perceived. H.R. Rajabi et al [13] applied a simple and efficient chemical precipitation method for the synthesis of pure and iron doped zinc sulfide quantum dots as novel semiconductor nanoparticles and malachite green was degraded by photo-catalytic process. Zhang et al[14] synthesized highly ordered $\mathrm{TiO}_{2}$ nanotube arrays $\left(\mathrm{TiO}_{2}-\mathrm{NTAs}\right)$, with a uniform tube size on titanium substrate by means of reoxidation and annealing and also fabricated a composite structure, by assembling CdSe quantum dots into $\mathrm{TiO}_{2}$-NTAs via cyclic voltammetry electrochemical deposition and its photo-catalytic activity is measured for methyl orange by varying the amounts of CdSe QDs. Furthermore, QDs get more and more attention because their confinement by the excited electrons and holes leads to optical and electronic properties different from those in bulk semiconductors [15]. Moreover, QDs as new semiconductor particles in the nanosized scales possess a higher surface area-to-volume ratio than their bulk counterparts, and thus allows for greater photon absorption on the photo-catalyst surface [16]. Furthermore, recombination of the electron-hole pair within the semiconductor particle drastically reduces as particle size decreases [17]. Therefore, the nanoscale semiconductor is expected to have higher photo-catalytic activity than its bulk. This material has been proved as a better photo-catalyst due to rapid generation of electron-hole pairs with photo-excitation as it is a direct wide band gap semiconductor material [18]. Furthermore, it possesses high negative reduction potential of excited electrons due to its higher conduction band (CB) position in aqueous solution as compared to other extensively studied photo-catalysts.

Metal-organic frameworks (MOFs), also known as nanoporous coordination networks, are highly ordered, that are derived from metallic centers bonded by terminal organic linkers [19]. MOFs have attracted a great deal of attention in both biological and industrial areas due to their numerous potential applications in the fields of gas storage [20], sensing [21], drug delivery [22], and catalysis [23]. In addition to these attractive applications, MOFs are active structures having high porosity [24] yielding large internal surface relatively and thereby facilitating enhanced catalytic reactivity [25]. If a MOF is employed as light-sensitive semiconductor, the central metal-oxide cluster can be considered as a discrete QD, which is stabilized and interconnected by the organic linkers acting as photon antenna [26]. Compared with traditional semiconductor photo-catalysts, the superiority of MOFs originates from their ultrahigh surface area and narrow micropore distribution, which may lead to the formation of mono-disperse photoactive species supported on MOFs [27-28]. However, the photo-activity of MOF was not as effective as that of inorganic semiconductor NPs. Using semiconductor QDs and MOFs as photo-catalysts have their own advantages and disadvantages [29]. By integrating such semiconductor QDs-MOF hybrid materials, they are expected to possess advanced properties originating from the two different components and also overcome their individual shortages [30-32]. To date, there has been progress on the studies of semiconductor- MOF heterostructures for photo-catalytic activity.

For the assessment of photo-catalytic activity, Phloxine B dye was preferred because of its intense use in biological applications such as antimicrobial substance [33], industrial applications [34] etc. Phloxine B dye is extensively used in different applications but the literature on the research of its degradation is inadequate [35-36]. Mir et al [35] proved that heterogeneous photo-catalysis using $\mathrm{TiO}_{2}$ as photo-catalyst to be an effective method for the decolorization of Phloxine B.

Here, we demonstrated the synthesis of CdSe quantum dots (QDs) sensitized Zr-1,3,5-benzene tricarboxylic acid (ZrBTC) metal-organic frameworks (MOFs) hetero-structure. CdSe QDs having small band gap therefore absorbing visible light illumination served their better photo-catalytic activity. The photo-catalytic degradation of Phloxine B dye using CdSe QDs-ZrBTC heterostructure is discussed in detail in this paper.

\section{Experimental Section:-}

\section{Chemicals:-}

All the chemicals were purchased from Sigma-Aldrich and the details are as follows. Cadmium Acetate $\left(\mathrm{Cd}\left(\mathrm{CH}_{3} \mathrm{CO}_{2}\right)_{2}, 99 \%\right)$, Sodium sulphite $\left(\mathrm{Na}_{2} \mathrm{SO}_{3}, 99 \%\right)$, Selenium (Se, 98\%), Tartaric acid $\left(\mathrm{C}_{4} \mathrm{H}_{6} \mathrm{O}_{6}, 99 \%\right)$, Triethanolamine $\left(\mathrm{C}_{5} \mathrm{H}_{15} \mathrm{NO}_{3}, \geq 99 \%\right)$, Sodium hydroxide $(\mathrm{NaOH}, 99 \%)$, Mercaptopropanoic acid $\left(\mathrm{HSCH}_{2} \mathrm{CH}_{2} \mathrm{CO}_{2} \mathrm{H}\right.$, $\geq 99 \%)$, Zirconium nitrate $\left(\mathrm{Zr}\left(\mathrm{NO}_{3}\right)_{4}, 99 \%\right)$, Benzene-1,3,5-tricarboxylic acid $\left(\mathrm{H}_{3} \mathrm{BTC}, 99 \%\right)$, Methanol(98\%), Acetone(99.8\%), Toluene (99.8\%), Ethanol (98\%), and Distilled water (18.3 $\Omega$ ). 


\section{Synthesis of ZrBTC:-}

$\mathrm{Zr}-\mathrm{MOF}$ was synthesized by the following procedure. First, $\mathrm{Zr}\left(\mathrm{NO}_{3}\right)_{4}(80 \mathrm{mg})$ was dissolved in distilled water (10 $\mathrm{ml})$ under stirring. Further, Benzene-1,3,5-tricarboxylic acid $\left(\mathrm{H}_{3} \mathrm{BTC}, 99 \%\right)(40 \mathrm{mg})$ in ethanol $(5 \mathrm{ml})$ solution was prepared and added the above solution at vigorous stirring at room temperature for the period of $6 \mathrm{~h}$. A white colour precipitate was formed and separated by centrifugation at $4000 \mathrm{rpm}$ and further washed with ethanol several times. The final product was dried in an oven at $90{ }^{\circ} \mathrm{C}$ under vacuum overnight and allowed for characterization.

\section{Synthesis of CdSe QDs:-}

The CdSe QDs were synthesized using green synthesis employing chemical bath deposition (CBD) method [37]. Here the sources of $\mathrm{Cd}$ and $\mathrm{Se}$ are Cadmium acetate $(\mathrm{CdAc})$ and sodium seleno sulphate $\left(\mathrm{Na}_{2} \mathrm{SeSO}_{3}\right)$ respectively. First, $10 \mathrm{~g}$ of sodium sulphite $\left(\mathrm{Na}_{2} \mathrm{SO}_{3}\right)$ was dissolved in $50 \mathrm{ml}$ of deionised water at room temperature (RT) until the solution became clear. To this solution, $0.5 \mathrm{~g}$ of elemental selenium was added and refluxed at $65^{\circ} \mathrm{C}$ for $6 \mathrm{~h}$. On other hand, $2.66 \mathrm{~g}$ of CdAc was dissolved in $50 \mathrm{ml}$ of deionised water. To this, $8 \mathrm{ml}$ of $1 \mathrm{M}$ Tartaric acid (TA) and $10 \mathrm{ml}$ of Triethanolamine (TEA) was added until the $\mathrm{pH}$ reaches to 8. Later, the solutions CdAc + TA+TEA and $\mathrm{Na}_{2} \mathrm{SeSO}_{3}$ were mixed in $1: 1 \mathrm{v} / \mathrm{v}$ ratio and heated at $65^{\circ} \mathrm{C}$ for $1 \mathrm{~h}$. In order to control the $\mathrm{pH}, 4 \mathrm{ml}$ of $5 \mathrm{M} \mathrm{NaOH}$ solution was added to the above solution. The resulted CdSe QDs were washed with methanol-acetone (v/v-1:2) solutions by centrifugation at $8000 \mathrm{rpm}$ and then stored in toluene.

\section{Synthesis of CdSe QDs-ZrBTC:-}

MPA capped CdSe QDs were prepared by the exchange of TA on the surface of QDs. For this, TA and TEA capped CdSe were washed with ethanol and water by centrifugation and stored in toluene. $100 \mathrm{mg}$ of ZrBTC (MPA) mixed in $5 \mathrm{ml}$ of ethanol and stirred for $2 \mathrm{~h}$ and to this, $50 \mathrm{mg}$ CdSe QDs are added. A pale-red color precipitate was formed. A pale-red color powder was obtained after solvent evaporation. The product was washed in water-ethanol $(2 \times 10 \mathrm{ml})$ solution several times by centrifugation at $8000 \mathrm{rpm}$ to remove the impurities and dried in oven at $90^{\circ} \mathrm{C}$. The resulted powder allowed characterizations to verify the CdSe QDs-ZrBTC structure.

\section{Characterization:-}

The phase and crystallinity of prepared samples were recorded on the X-ray diffraction technique (XRD, Bruker, D8-Advanced, Germany) with $\mathrm{Cu}-\mathrm{K} \alpha$ irradiation in the range of $2 \theta$ from $10^{\circ}$ to $80^{\circ}$. The optical properties of prepared samples were investigated using an ultraviolet visible spectrophotometer (UV-Vis, Speccord 200 plus, range 200-1000 nm). The prepared samples were sputter coated with Au for $100 \mathrm{~s}$ and the morphology was examined using a field emission scanning electron microscopy (FESEM, Carl Zeiss SUPRA 55VP, Germany). The surface morphology variations of CdSe QDs and ZrBTC composites are analysed by HRTEM. Lattice fringes and SAED patterns of synthesized powders were examined using high resolution transmission electron microscope (HRTEM, FEI make, Tecnai F20 S-TWIN TMP, Netherlands) with $0.144 \mathrm{~nm}$ resolution. Photo-catalytic dye degradation experiments were carried out with $300 \mathrm{~W}$ Xe -lamp. The variations in dye degradation are measured UV-visible spectrometer. Dye degradation \% is calculated as per following equation

Dye degradation $\%=\left\{\left(\mathrm{C}_{0}-\mathrm{C}\right) / \mathrm{C}_{0}\right\} * 100$

Where, $\mathrm{C}_{0}$ is initial concentration and $\mathrm{C}$ is final concentration.

The photo-catalyst is recycled as per follows. After the completion of initial dye degradation experiment, the total solution was centrifuged at $4000 \mathrm{rpm}$ and the photo-catalyst is separated and washed with ethanol-water solution and dried. The recycled catalyst weight is measured and used further dye degradation experiments.

\section{Results and Discussion:-}

\section{X-ray diffraction Analysis:-}

The nature of phase purity and crystallinity of CdSe QDs and ZrBTC MOFs are examined using x-ray diffraction analysis and presented in Figure 1a and 1b. In figure 1a, the planes (111), (220) and (311) corresponds to CdSe semiconductor QDs and demonstrate crystalline cubic phase for CdSe QDs , which have strong match with reported procedures[38]. In figure $1 \mathrm{~b}$, the sharp peaks for ZrBTC at $2 \theta$ values of $17.26^{0}, 19.11^{0}, 25.53^{\circ}, 27.23^{0}$, $29.06^{0}, 34.89^{\circ}$ and $40.42^{\circ}$ demonstrate high crystallinity and bulk phase of ZrBTC MOFs are well attached with previous reports[39]. The broad spectrum indicates nanosize structures of MOFs. 


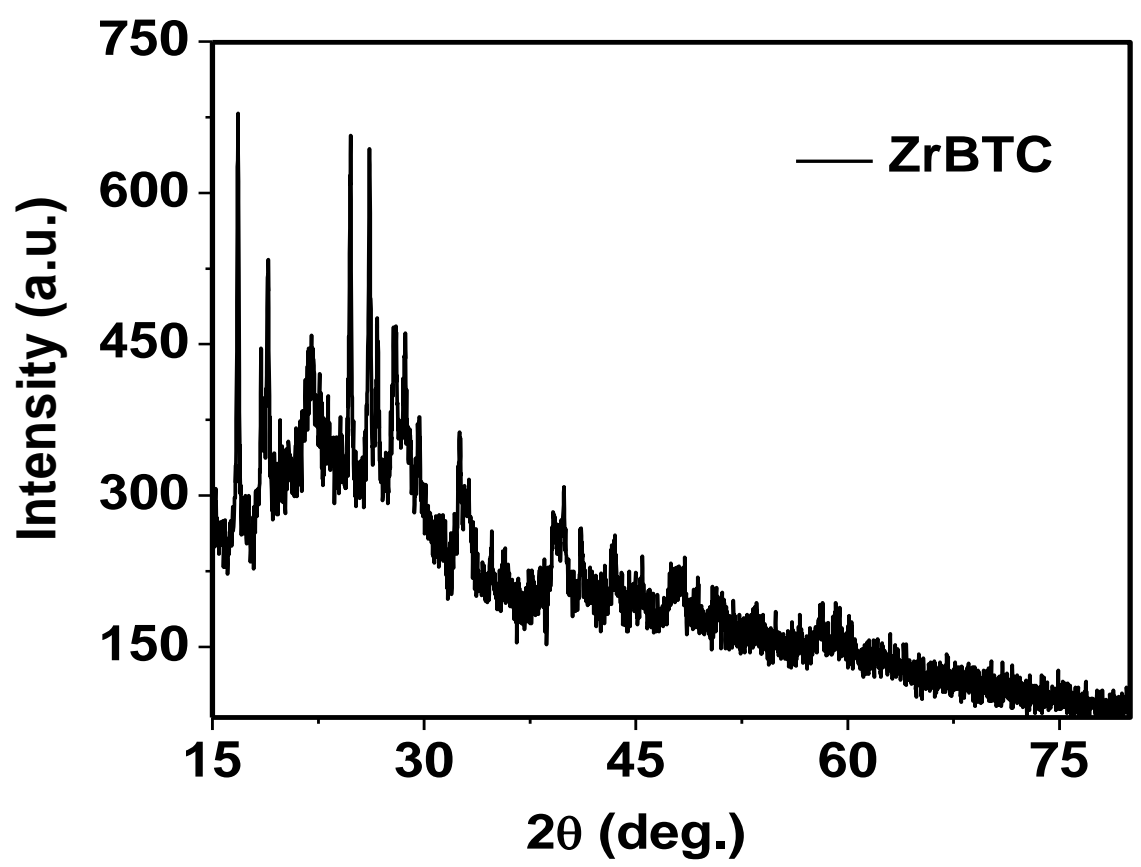

Figure 1a:- X-ray diffraction spectrum of ZrBTC metal-organic frameworks.

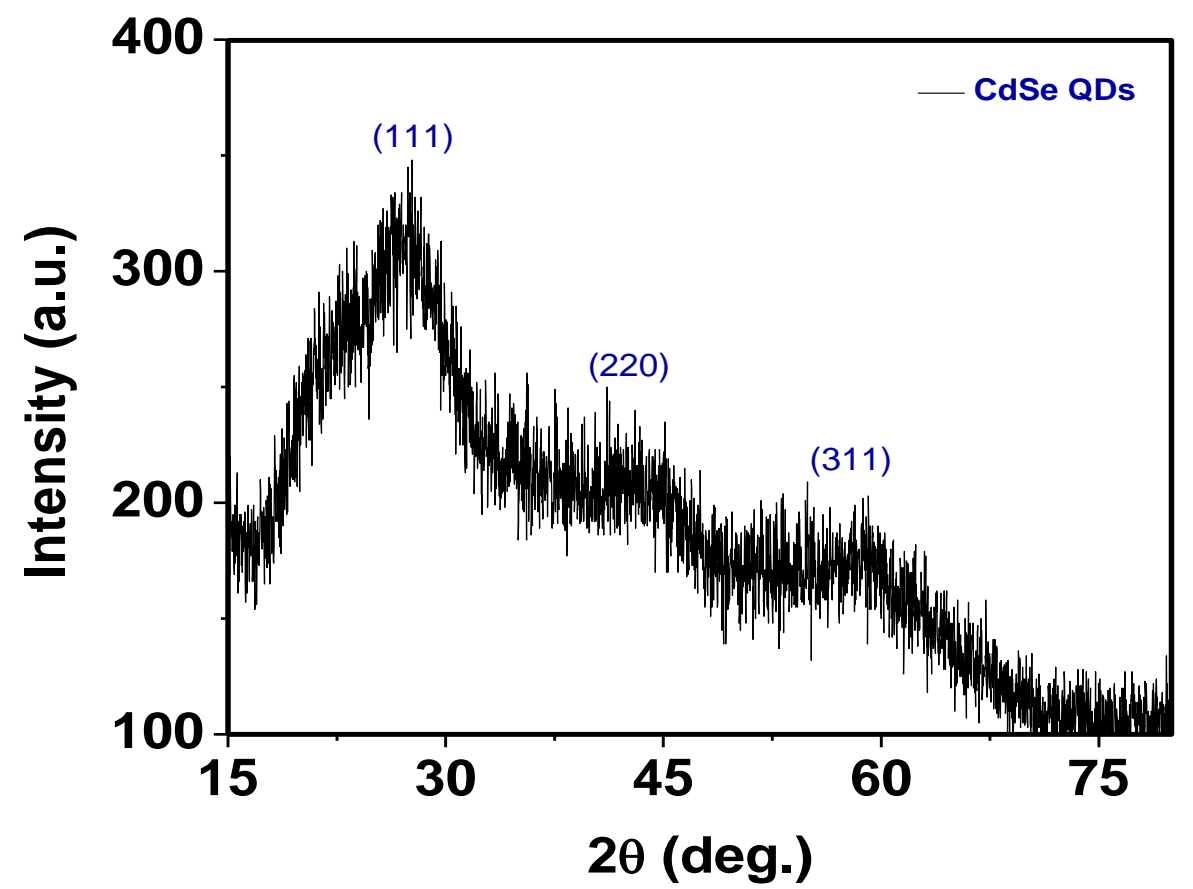

Figure 1b:- X-ray diffraction spectrum of CdSe QDs.

Surface morphological observations:-

FESEM:-

The surface morphological observations of ZrBTC MOFs are presented in Figure 2. Figure 2 show that $\mathrm{ZrBTC}$ MOFs formed into 2D sheets. 


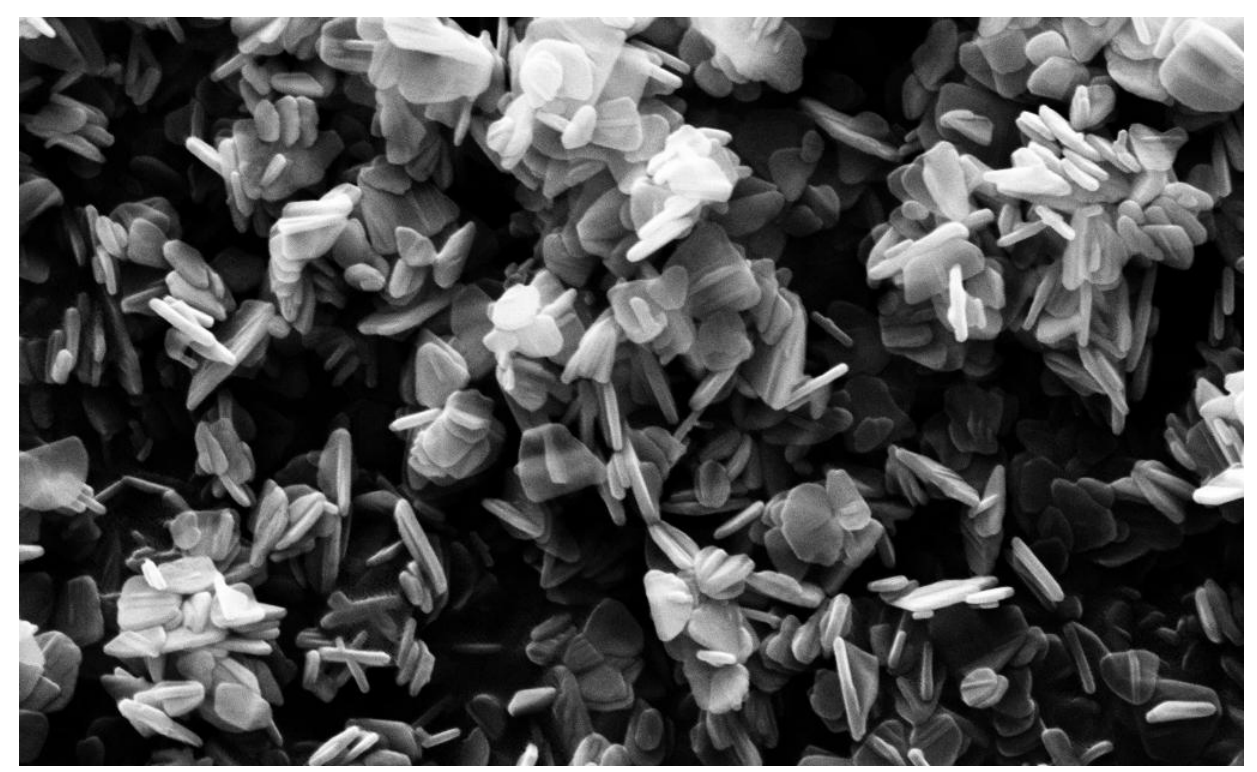

Figure 2:- FESEM image represents the surface morphology of ZrBTC.

\section{HRTEM:-}

The transition electron microscopy images of CdSe QDs-ZrBTC compound are shown in Figure 3. As we observed in Figure 3a, the structure of MOFs is 2D sheets. Figure 3b shows the presence of CdSe QDs and ZrBTC MOFs. Figure $3 \mathrm{c}$ represents the surface morphology of CdSe semiconductor quantum dots and the corresponding SAED pattern is shown in Figure 3d. From the Figure 3, it is observed that MOFs are formed as 2D sheets and CdSe semiconductor quantum dots are in spherical shape. In Figure 3b, it is clearly seen that the CdSe semiconductor quantum dots are sensitized on ZrBTC MOFs. 


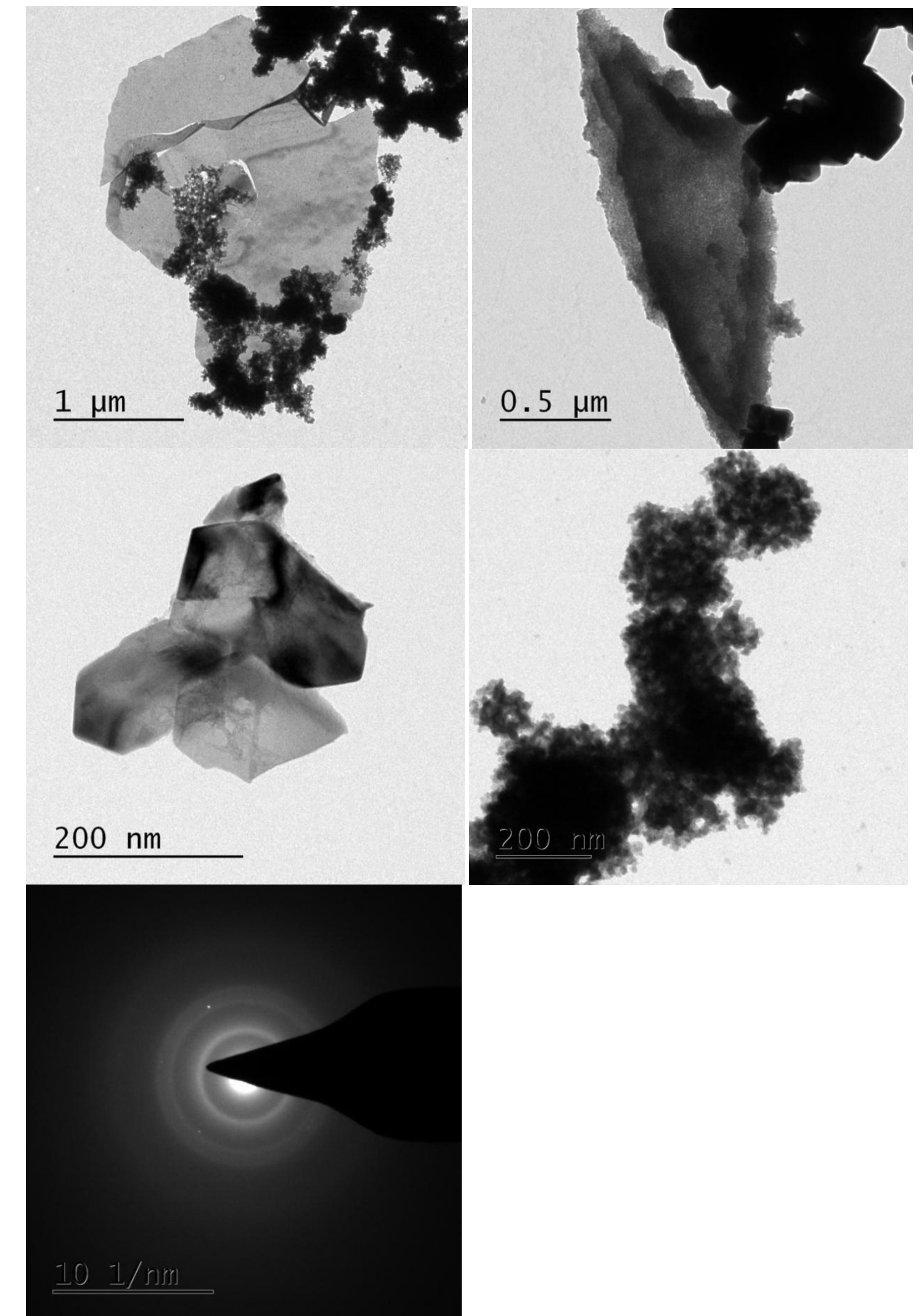

Figure 3:- HRTEM images of CdSe QDs-ZrBTC(a\&b), (c) ZrBTC (d) CdSe semiconductor quantum dots and (e) SAED. 


\section{UV-visible spectroscopic Studies:-}

The UV-visible spectrum of CdSe QDs sensitized ZrBTC indicates the absorption peaks at $520 \mathrm{~nm}$. The sharp absorption peak below $300 \mathrm{~nm}$ in the UV-visible spectrum of ZrBTC demonstrates that pure ZrBTC MOFs have an absorption in the UV light region. The broad absorption peak at $520 \mathrm{~nm}$ is imputed to absorbance spectra of ZrBTC MOFs and the corresponding QDs-sensitized MOFs proclaiming the visible light absorption properties of CdSe semiconductor quantum dots. As shown, absorption edge for CdSe QDs-ZrBTC heterostructure shifts to longer wavelength, indicating the visible light absorption of CdSe. Comparatively, after deposited with CdSe, the heterostructures exhibited higher light absorption in visible light regions. These results indicate that the deposition of CdSe extended the absorption of the CdSe QDs-ZrBTC into the visible light region. The ordered ZrBTC MOFs have interior surfaces on which CdSe nanoparticles can be deposited, resulting in an enhancing absorption capacity in the visible light region while collecting and transmitting electrons through the $\mathrm{ZrBTC}$. The formation of $\mathrm{CdSe}$ QDs-ZrBTC hetero-structure compound is also revealed here.

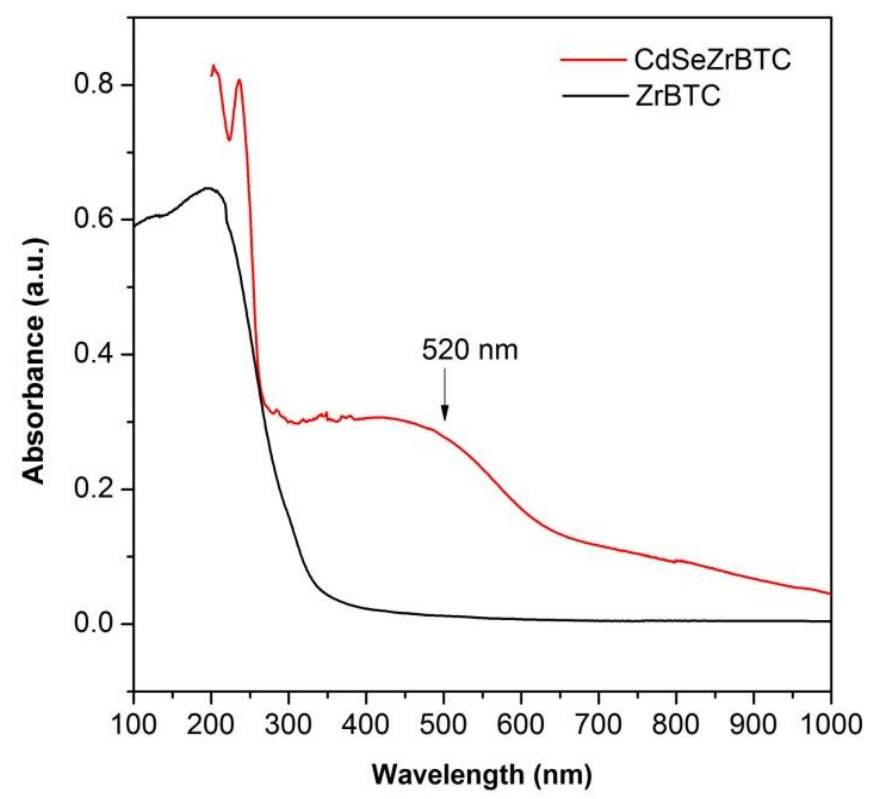

Figure 4:- UV-visible spectrum of CdSe QDs sensitized ZrBTC and ZrBTC

\section{Photo-catalytic dye degradation studies:-}

The photo-catalytic activity of the CdSe QDs-ZrBTC hetero-structure was operated using a xenon lamp (300 W) at room temperature as reported in literature [40]. The experiments were carried out in a $250 \mathrm{ml}$ capacity beaker at ambient temperature between $25^{\circ} \mathrm{C}$ and $30^{\circ} \mathrm{C}$. In each experiment, $100 \mathrm{mg}$ samples were measured into $20 \mathrm{~mL}$ of Phloxine B solution (initial concentration, $\mathrm{C}_{\mathrm{o}}=10 \mathrm{mgL}^{-1}$ ). Prior to irradiation the suspension was stirred for $30 \mathrm{~min}$ in the dark to attain adsorption-desorption equilibrium. Then the concentration of dye in the solution was measured and no change in the concentration was perceived here. The degradation of Phloxine B was evaluated in the presence of CdSe QDs-ZrBTC under visible light illumination. Regulated experiments were operated to confirm that Phloxine B was not photo-degraded without photo-catalyst in the dark. Figure 5(a) interprets the time dependent UV-Vis spectra for Phloxine B dye degradation curves of CdSe QDs-ZrBTC MOFs under visible light illumination. The relative intensity of the maximum absorbance at $550 \mathrm{~nm}$ in UV-Vis spectra evaluates the amount of Phloxine B dye degradation over the surface of CdSe QDs-ZrBTC heterostructure. The intensity of UV absorbance of Phloxine $\mathrm{B}$ dye molecules decreases continuously with the increase of time interval from $0 \mathrm{~min}$ to $100 \mathrm{~min}$, evincing the degradation of Phloxine B dye over the surface of CdSe QDs-ZrBTC. Figure 5(b) accentuates the concentration changes of Phloxine B concentration $\left(\mathrm{C} / \mathrm{C}_{0}\right)$ in the presence and absence of photo-catalyst with increase of photodegradation time. A separate catalytic experiment is performed under dark condition for $60 \mathrm{~min}$ in order to confirm any external effect on the Phloxine $\mathrm{B}$ degradation. This shows the negligible decrease in the concentration of Phloxine B dye. Furthermore, the Phloxine B degradation without catalyst shows very low degradation within the time interval of $100 \mathrm{~min}$, indicating that the Phloxine B dye cannot degrade by itself under light illumination. The relative concentration $\left(\mathrm{C} / \mathrm{C}_{0}\right)$ of Phloxine $\mathrm{B}$ dye molecules decreases with the enhanced time interval of 0 min to 100 
min in the presence of CdSe QDs-ZrBTC catalyst demonstrating the better photo-catalytic dye degradation of Phloxine B.
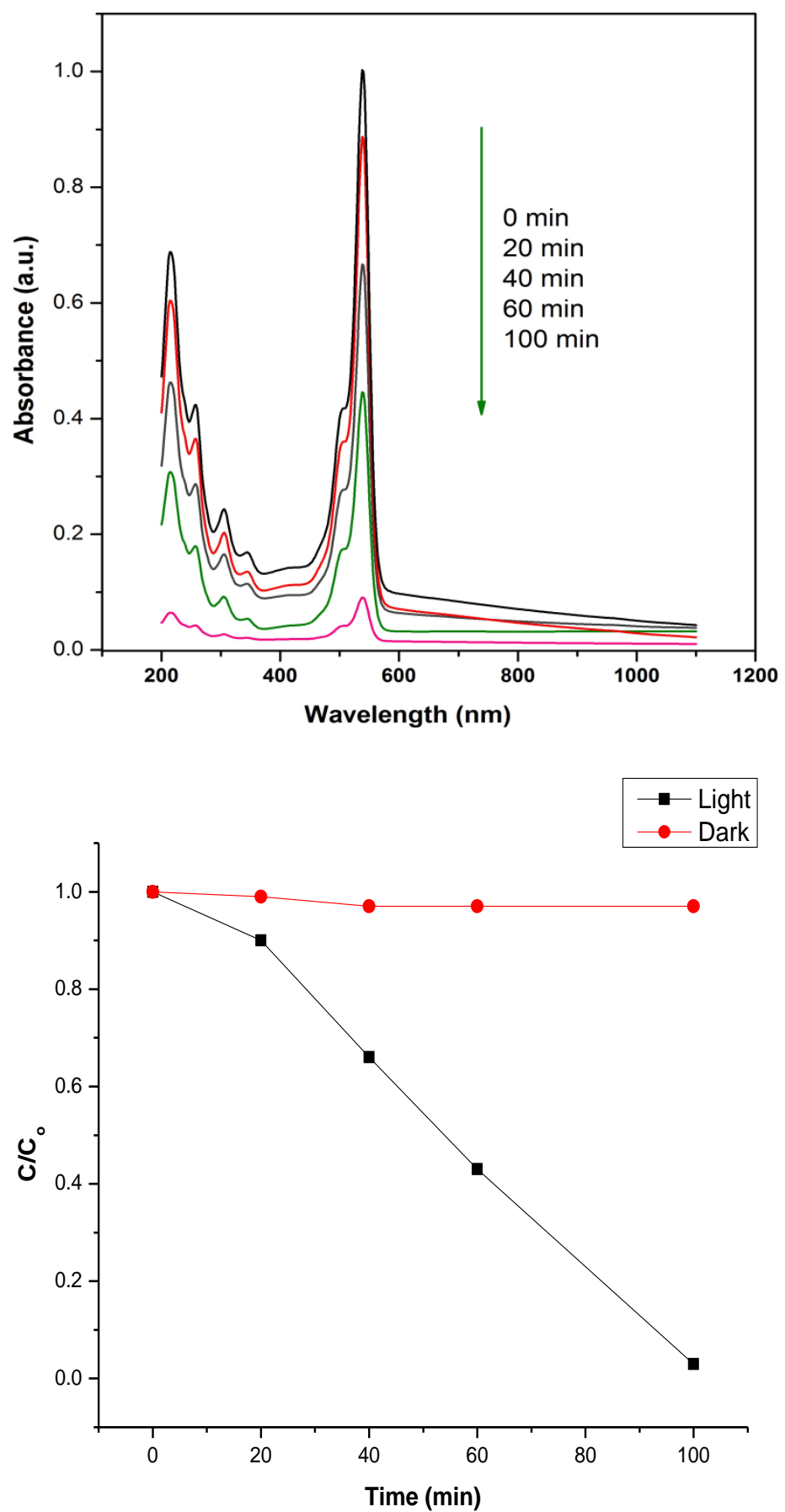

Figure 5:- Time dependent UV-vis spectra for Phloxine B dye degradation curves of CdSe QDs-ZrBTC MOFs under visible light illumination (a). Photo-degradation curves of Phloxine B dye with respect to irradiation time (b)

\section{Photo-catalytic Mechanism:-}

The Photo-catalytic mechanism of CdSe QDs-ZrBTC for the degradation of Phloxine B dye was investigated here. The CdSe QDs have a relatively small band gap, and therefore have the potential to make use of visible light in the solar spectrum. CdSe quantum dots have strong fluorescence in the visible region. The 2D ZrBTC MOFs provide 
platform for CdSe QDs and hinder the effective electron-hole pair recombination under photo-excitation. Fluorescence is the emission of a photon from a molecule as an electron goes from an excited state to a ground state. The color of the emission depends on the energy difference between states. The light can be absorbed efficiently by both the QD and the pollutant molecule (P). According to the proposed pathway for the photo-catalytic activity of the QDs, when the irradiation energy exceeds the energy difference between the valence and conduction bands (band gap) of a semiconductor, electrons in the valence band of QDs could be excited to the conduction band and electron-hole pairs are generated (Eq. (1)).

$\mathrm{CdSe} \mathrm{QDs}+\mathrm{h} v \rightarrow \mathrm{CdSe}$ QDs $\left(\mathrm{e}^{-}\right)+\mathrm{CdSe} \mathrm{QDs}\left(\mathrm{h}^{+}\right)$

This is referred to as the photo-excited state of the semiconductor. The photo-generated charges (i.e., $\mathrm{e}^{-}$and $\mathrm{h}^{+}$ carriers) cause oxidation reactions on the particle surface, giving rise to free radicals, which in turn degrade the organic molecules. Then the molecular oxygen $\left(\mathrm{O}_{2}\right)$ near the interface photo-catalyst could be quickly reduced to the superoxide radical $\left(\mathrm{O}_{2} \bullet^{-}\right)$and hydrogen peroxide radical $(\cdot \mathrm{OOH})$ by the photo-generated electrons (Eqs. (2) - (5)), whereas the valence band holes can directly oxidize organic pollutants adsorbed on the surface of catalyst (QDs) or mineralized them indirectly through hydroxyl radicals $(\bullet \mathrm{OH})$ generated by the reaction of holes and water molecules $\left(\mathrm{H}_{2} \mathrm{O}\right)$ or chemisorbed $\left(\mathrm{OH}^{-}\right)$(Eqs. $\left.(6)-(8)\right)$ [12-14]. Then, under visible-light irradiation, some photo-generated electrons can transfer from excited-state of adsorbed molecules to the conduction band (CB) of QDs and can reduce molecular oxygen to superoxide anion, then the superoxide anion can react with $\mathrm{H}_{2} \mathrm{O}$ to form other photo-induced active species (e.g., $\left.\mathrm{O}_{2} \bullet^{-}, \bullet \mathrm{OOH}, \bullet \mathrm{OH}\right)$ to oxidize or degrade the surface-absorbed organic pollutants.

$\mathrm{QDs}\left(\mathrm{e}^{-}\right)+\mathrm{O}_{2} \rightarrow \mathrm{QDs}+\mathrm{O}_{2}^{\cdot-}$

$\mathrm{O}_{2}{ }^{--}+\mathrm{H}^{+} \rightarrow \mathrm{HO}_{2}{ }^{-}$

$2 \mathrm{HO}_{2} \cdot \rightarrow \mathrm{H}_{2} \mathrm{O}_{2}+\mathrm{O}_{2}$

$\mathrm{H}_{2} \mathrm{O}_{2}+\mathrm{e}^{-} \rightarrow \mathrm{OH}^{-}+\mathrm{OH}^{\cdot}$

$\mathrm{QDs}\left(\mathrm{h}^{+}\right)+\mathrm{H}_{2} \mathrm{O} \rightarrow \mathrm{QDs}+\mathrm{HO}^{\cdot}+\mathrm{H}^{+}$

$\mathrm{QDs}\left(\mathrm{h}^{+}\right)+\mathrm{OH}^{-} \rightarrow \mathrm{QDs}+\mathrm{OH}^{-}$

QDs $\left(\mathrm{h}^{+}, \mathrm{O}_{2}{ }^{\cdot-},{ }^{\cdot} \mathrm{OOH},{ }^{\cdot} \mathrm{OH}\right)+$ Phloxine $\mathrm{B} \rightarrow$ Products

On the other hand, because of CdSe QDs sensitized ZrBTC, a strong band exists between the CdSe QDs and the surface groups of ZrBTC. Thus the excited electrons can be effectively used to obtain more super oxide ions, increasing the photo-degradation rate.Therefore the holes, electrons, hydroxyl radicals, super oxide radicals and oxygen play an important role in the photo-catalytic reaction mechanism. Therefore, the produced hydroxyl radicals can cause organic matter mineralization.

In our case, the remarkable photo-catalytic activity of CdSe QDs sensitized with ZrBTC is due to the efficient transfer of the energy from absorbed photons to the impurity, quickly localizing the excitation and suppressing undesirable reactions on the nanocrystal surface. In the present paper the Phloxine B dye has effectively degraded by $97 \%$ within $100 \mathrm{~min}$.

\section{Conclusion:-}

The visible light effectual CdSe QDs and 2D ZrBTC sheets are synthesized in a multistep procedure and demonstrated the augmented photo-catalytic degradation studies of Phloxine B dye under visible light illumination.

Author Information:-

Corresponding Author:

Email: haritha.gonthina@gmail.com

Notes: The authors don't have any competing financial interest

\section{Acknowledgements:-}

We acknowledge to the department of engineering chemistry, AUCE (A), Andhra university for providing general lab facilities and technical support. 


\section{References:-}

1. Gopal, K.V. and Wynkoop, D. (1996): Environmental Photochemistry on Semiconductor Surfaces: Photosensitized Degradation of a Textile Azo Dye, Acid Orange 7, on TiO2 Particles Using Visible Light. Environ. Sci. Technol.,30:1660-1666

2. Adegoke, K.A. and Bello, O.S.(2015): Dye sequestration using agricultural wastes as adsorbents. Water Resources and Industry, 12: 8-24.

3. Assadi, A., Nateghi, R., Bonyadinejad, G.R. and Amin, M.M.(2012): Application of coagulation process reactive blue 19 dye removal from textile industry wastewater .International Journal of Environmental Health Engineering, 1: 1-5.

4. Moosvi, S., Keharia, H. and Madamwar, D. (2005): Decolourization of textile dye Reactive Violet 5 by a newly isolated bacterial consortium RVM 11.1. World Journal of Microbiology \& Biotechnology , 21:667-672

5. Mukhlish, M. Z. B., Najnin, F., Rahman, M. M. and Uddin, M. J. (2013): Photocatalytic Degradation of Different Dyes Using $\mathrm{TiO}_{2}$ with High Surface Area: A Kinetic Study. J. Sci. Res., 5: 301-314.

6. Neppolian, B., Sakthivel, S., Arabindoo, B., Palanichamy, M. and Murugesan, V. (1999): Degradation of textile dye by solar light using $\mathrm{TiO}_{2}$ and $\mathrm{ZnO}$ photo-catalysts. J. Environ. Sci. Health, A, 34: 1829-1838.

7. Dafare, S., Deshpande, P .S. and Bhavsar, R. S. (2013): Photocatalytic Degradation of Congo Red dye on Combustion synthesized $\mathrm{Fe}_{2} \mathrm{O}_{3}$. Indian Journal of Chemical Technology, 20: 406-410.

8. Luo, J. and Hepel, M. (2001): Photoelectrochemical degradation of naphthol blue black diazo dye on $\mathrm{WO}_{3}$ film electrode. Electrochimica Acta, 46: 2913-2922.

9. Periyat, P., Pillai, S. C., McCormack, D. E., Colreavy, J. Steven, J. (2008): Hinder Improved High-Temperature Stability and Sun-Light-Driven Photocatalytic Activity of Sulfur-Doped Anatase $\mathrm{TiO}_{2}$. J. Phys. Chem. C, 112: 7644-7652.

10. Kruanetr, S., Tan-arsa, N. and Wanchanthuek, R. (2013): The Study of Methylene Blue Removal by Using Mixed $\mathrm{TiO}_{2}$ as a Catalyst under Solar Light Irradiation. International Journal of Scientific and Research Publications, 3: 1-7.

11. Zhang, Y.G ., Ma, L.L., Li , J.L. and Yu, Y . (2007): In Situ Fenton Reagent Generated from $\mathrm{TiO}_{2} / \mathrm{Cu}_{2} \mathrm{O}$ Composite Film: a New Way to Utilize $\mathrm{TiO}_{2}$ under Visible Light Irradiation Environ. Sci. Technol., 41: 6264-6269.

12. Muthulingam, S., Lee, I.H. and Uthirakumar, P. (2015): Highly efficient degradation of dyes by carbon quantum dots/N-doped zinc oxide $(\mathrm{CQD} / \mathrm{N}-\mathrm{ZnO})$ photocatalyst and its compatibility on three different commercial dyes under daylight. Journal of Colloid and Interface Science, 455: 101-109.

13. Rajabi , H. R. , Khani , O. , Shamsipur, M. and Vatanpour, V. (2013): High-performance pure and $\mathrm{Fe}^{3+}$-ion doped $\mathrm{ZnS}$ quantum dots as green nanophotocatalysts for the removal of malachite green under UV-light irradiation. Journal of Hazardous Materials, 250-251: 370-378.

14. Zhang, M., Xu, Y., Lv, J., Yang, L., Jiang, X., He, G., Song, X. and Sun, Z. (2014): Capability of coupled $\mathrm{CdSe} / \mathrm{TiO}_{2}$ heterogeneous structure for photocatalytic degradation and photoconductivity. Nanoscale Research Letters, $9: 1-7$.

15. Chen, C., Zhai, Y. , Li, C. and Li, F. (2014): Improving the efficiency of cadmium sulfide-sensitized titanium dioxide/indium tin oxide glass photoelectrodes using silver sulfide as an energy barrier layer and a light absorber. Nanoscale Research Letters, 9:1-9.

16. Ke, F., Wang, L. and Zhu. J. (2015): Facile fabrication of CdS-metal-organic framework nanocomposites with enhanced visible-light photocatalytic activity for organic transformation. Nano Research, 8: 1834-1846.

17. Zhang, H. , Ming, H., Lian, S., Huang, H., Li, H. , Zhang, L., Liu, Y. , Kang ,Z. and Leeb, S.T. (2011): $\mathrm{Fe}_{2} \mathrm{O}_{3} /$ carbon quantum dots complex photocatalysts and their enhanced photocatalytic activity under visible light. Dalton Trans., 40:10822-10825.

18. Zhang, H. , Huang, H., Ming, H. , Li, H. , Zhang, L. , Liu, Y. and Kang, Z. (2012): Carbon quantum dots $/ \mathrm{Ag}_{3} \mathrm{PO}_{4}$ complex photocatalysts with enhanced photocatalytic activity and stability under visible light. J. Mater. Chem., 22: 10501-10506.

19. Ragon, F., Campo, B., Yang, Q., Martineau, C., Wiersum, A. D. , Lago, A. , Guillerm, V. , Hemsley, C.,Eubank, J. F. , Vishnuvarthan, M., Taulelle, F. , Horcajada, P.,Vimont, A. , Llewellyn, P.L. , Daturi, M. , Vinot, S. D.Maurin, G. , Serre, C., Devic, T. and Clet, G. (2015): Acid-functionalized UiO-66(Zr) MOFs and their evolution after intra-framework cross-linking: structural features and sorption properties. J. Mater. Chem. A, 3:3294-3309.

20. Eddaoudi, M. , Li, H. and Yaghi, O. M. (2000): Highly Porous and Stable Metal-Organic Frameworks: Structure Design and Sorption Properties. J. Am. Chem. Soc., 122: 1391-1397. 
21. Allendorf, M.D. , Houk, R. J. T. , Andruszkiewicz, L., Talin, A. A. , Pikarsky, J. , Choudhury, A. , Gall, K. A. and Hesketh, P. (2008): J. Stress-Induced Chemical Detection Using Flexible Metal-Organic Frameworks. J. AM. CHEM. SOC., 130: 14404-14405.

22. McKinlay, A. C., Morris, R.E. , Horcajada, P., Frey, G., Gref, R. , Couvreur, P. and Serre, C. (2010): MetalOrganic Frameworks in Biomedicine. Angew. Chem. Int. Ed., 49:6260 - 6266.

23. Luz, I., Xamena F.X. L. and Corma, A. (2012): Bridging homogeneous and heterogeneous catalysis with MOFs: $\mathrm{Cu}-\mathrm{MOF}$ as solid catalysts for three-component coupling and cyclization reactions for the synthesis of propargylamines, indoles and imidazopyridines. Journal of Catalysis, 285: 285-291.

24. Butler, K. T. , Hendon, C. H., and Walsh, A. (2014): Electronic Chemical Potentials of Porous Metal-Organic Frameworks. J. Am. Chem. Soc., 136: 2703-2706.

25. Wu, C.D. , Hu, A. , Zhang, L. and Lin, W. (2005): A Homochiral Porous Metal-Organic Framework for Highly Enantioselective Heterogeneous Asymmetric Catalysis. J. AM. CHEM. SOC., 127:8940-8941.

26. Xamena, F. X. L., Corma, A. and Garcia, H. (2007): Applications for Metal-Organic Frameworks (MOFs) as Quantum Dot Semiconductors. J. Phys. Chem. C, 111: 80-85.

27. Amador, R. N. , Carboni, M. and Meyer, D. (2017): Sorption and photodegradation under visible light irradiation of an organic pollutant by a heterogeneous UiO-67-Ru-Ti MOF obtained by post-synthetic exchange. RSC Adv., 7:195-200.

28. Shi , L., Wang, T. , Zhang, H. , Chang, K. , Meng , X., Liu , H. and Ye, J. (2015): An Amine-Functionalized Iron(III) Metal-Organic Framework as Effi cient Visible-Light Photocatalyst for Cr(VI) Reduction. Adv. Sci., 2:1500006

29. Yang, H., He, X. W., Wang, F., Kang, Y. and Zhang, J. (2012): Doping copper into ZIF-67 for enhancing gas uptake capacity and visible-light-driven photocatalytic degradation of organic dye. J. Mater. Chem., 22: 2184921851

30. Xu, X.Y. and Yan. B. (2017): Eu(III)-functionalized ZnO@MOF heterostructures: integration of preconcentration and efficient charge transfer for the fabrication of a ppb-level sensing platform for volatile aldehyde gases in vehicles. J. Mater. Chem. A, 5: 2215-2223.

31. Zhan, W., Kuang, Q. , Zhou, J., Kong, X., Xie, Z. and Zheng,L. (2013): Semiconductor@Metal-Organic Framework Core-Shell Heterostructures: A Case of ZnO@ZIF-8 Nanorods with Selective Photoelectrochemical Response. J. Am. Chem. Soc., 135:1926-1933.

32. Zhang, C.F., Qiu, L.G. , Ke, F. , Zhu, Y.J., Yuan, Y.P., Xua, G.S. and Jiang, X. (2013): A novel magnetic recyclable photocatalyst based on a core-shell metal-organic framework $\mathrm{Fe}_{3} \mathrm{O}_{4} @ \mathrm{MIL}-100(\mathrm{Fe})$ for the decolorization of methylene blue dye. J. Mater. Chem. A, 1:14329-14334.

33. Mihalyi, A., Jamshidi, S., Slikas, J. and Bugg, T. D. H. (2014): Identification of novel inhibitors of phosphoMurNAc-pentapeptide translocase MraY from library screening: Isoquinoline alkaloid michellamine B and xanthene dye phloxine B Bioorganic \& Medicinal Chemistry. 22 :4566-4571.

34. Keum, Y. S. , Kim, J.H. and Li, Q. X. (2003): Relationship between Singlet Oxygen Formation and Photolysis of Phloxine B in Aqueous Solutions. Journal of Photoscience, 10: 219-223.

35. Mir, N.A., Khan, A.,Kumar. and Muneer, M. (2013): Photocatalytic Study of a Xanthene Dye Derivative, Phloxine B in Aqueous Suspension of $\mathrm{TiO}_{2}$ : Adsorption Isotherm and Decolourization Kinetics. Energy and Environment Focus ,2 : 208-216.

36. Jain, R. and Shrivastava, M. (2008): Photocatalytic removal of hazardous dye cyanosine from industrial waste using titanium dioxide. Journal of Hazardous Materials, $152: 216-220$.

37. Oudhia, A. and Bichpuria, P. (2014): Green wet chemical route to synthesize capped CdSe quantum dots. Bull. Mater. Sci., 37:15-18.

38. Amiri, G. R. , Fatahian, S. and Mahmoudi. S. 2013: Preparation and Optical Properties Assessment of CdSe Quantum Dots. Materials Sciences and Applications, 4: 134-137.

39. Farhadia, S. , Manteghia, F. and Karimia. M. (2016): Application of a Zr-Metal Organic Framework in NCPE for Lithium Battery. Sciforum Electronic Conference Series, 20:1-30.

40. Cheng, C. , Fang, J. , Lu, S., Cen, C., Chen, Y., Ren, L. , Fenga, W. and Fanga, Z. (2016): Zirconium metalorganic framework supported highly-dispersed nanosized BiVO4 for enhanced visible-light photocatalytic applications. J Chem Technol Biotechnol., 91:2785-2792. 